\title{
Über die chemische Veränderung der Leber bei der Phosphorvergiftung. ${ }^{1}$ ) \\ Von
}

Alfred J. Wakeman.

(Aus dem physiologischen Institut zu Heidelberg.)

(Der Redaktion zugegangen am 12. März 1905.)

Während man früher bei der Erörterung des tierischen Stoffwechsels die in den Geweben vorhandenen oder in der Nahrung zugeführten Eiweißsubstanzen als ganze, ungeteilte Moleküle in die Rechnung einführte, hat die neuere Eiweißchemie eine andere Betrachtungsweise ermöglicht. Es ist mehr und mehr gelungen, die Zusammensetzung des Eiweißmoleküls aus einer ${ }^{\top}$ gewissen Zahl von organischen Verbindungen zu erkennen, die meistens zur Gruppe der Amidosäuren gehören. Diese Bestandteile besitzen in ihrem eigenen innern chemischen Gefüge eine bedeutende Festigkeit, sie lösen sich unter der Wirkung verschiedener Kräfte aus dem Verbande, der sie zum Proteinmolekül vereinigt, als widerstandsfähige Gruppen heraus. Es ist daher gerechtfertigt, mit ihnen als mit den Grundeinheiten des tierischen Stoffwechsels zu rechnen, und diese Anschauungsweise wird unzweifelhaft manche Vorteile darbieten. Sie ist um so mehr gerechtfertigt, als man diese Teile des Eiweißmoleküls auch wirklich in freiem Zustande in den Organismen vorfindet, z. B. in den keimenden Samen der Pflanzen und im Verdauungstraktus der Tiere. Da diese «Bausteine des Eiweißmoleküls» sich unter einander durch ihre chemische

ग) Vorläufige Mitteilung in der Berliner klinischen Wochenschrift, 1904, Nr. 41. 
Konstitution beträchtlich unterscheiden, so werden sie sich auch bei den physiologisch-chemischen Prozessen verschieden verhalten. Von den verschiedenen Teilen des Eiweißmoleküls wird der eine leichter in dieses, der andere leichter in jenes Stoffwechselprodukt übergehen.

Untersuchungen über die Beteiligung gewisser Bestandteile des Eiweißmoleküls an den Stoffwechselvorgängen werden zunächst hauptsächlich darin bestehen, daß man ihre Vermehrung oder Verminderung unter bestimmten physiologischen oder pathologischen Bedingungen feststellt.

Solche Feststellungen sind durch die neueren Arbeiten auf dem Gebiete der Eiweißchemie ermöglicht, welche uns für gewisse Glieder des Eiweißmoleküls, z. B. Histidin, Arginin, Lysin, Glykokoll und Tyrosin, hinreichende quantitative Bestimmungsmethoden gegeben haben.

Auf Veranlassung des Herrn Professor A. Kossel habe ich einige vergleichende Bestimmungen dieser Art ausgeführt, die sich freilich zunächst auf ein kleines Gebiet beschränken mußten. Ich untersuchte die Mengen bestimmter im Eiweiß enthaltener Atomgruppen, indem ich zunächst ganz davon absah, in welchem chemischen Zusammenhang die untersuchten Atomgruppen vorliegen, ob sie diesem oder jenem Eiweißkörper, diesem oder jenem morphotischen Bestandteil der Zelle angehören. Als Objekt der Analysen wurde Histidin, Arginin und Lysin ausgewählt, weil diese sich nach den Methoden von A. Kossel und seinen Mitarbeitern ziemlich leicht und genau bestimmen lassen.

Die Bestimmungen wurden an der Leber von Hunden ausgeführt und zwar einerseits im normalen Zustand dieses Organs, andererseits nachdem durch subakute Phosphorvergiftung ein Zerstörungsprozeß eingeleitet war. Es handelte sich also um die Frage, wie verhalten sich die genannten Bestandteile der Eiweißkörper bei einer krankhaften Veränderung, die nach Ausweis schon der älteren von Frerichs und Städeler gemachten Beobachtungen zu einer teilweisen Aufspaltung des Zelleneiweißes führt. Werden die genannten Basen schneller oder langsamer fortgeschafft als die übrigen Teile des Eiweiß- 
moleküls? Sind sie vorwiegend in denjenigen Teilen der Zelle lokalisiert, welche bei diesen Prozessen zuerst angegriffen und aufgelöst werden? Die folgenden Untersuchungen zeigen, daß dies letztere in der Tat der Fall ist.

\section{Versuchsanordnung.}

Für die Versuche dienten 5 Hunde mittlerer Größe, deren zwei (Nr. III und IV) im Laufe von 14 Tagen bis 3 Wochen durch subkutane Injektion von Phosphoröl vergiftet wurden.

Der Hund Nr. III erhielt am 13., 14., 15., 20. und 28. Juni, ferner am 1., 4., 6. und 9. Juli eine subkutane Injektion von $0,5 \mathrm{ccm}$ einer ziemlich starken Lösung von Phosphor in Olivenöl, am 11. VII. $0,6 \mathrm{ccm}$, am 12 . VII. $0,7 \mathrm{ccm}$, am 14 . VII. $0,7 \mathrm{ccm}$, am 15. VII. $0,8 \mathrm{ccm}$. An diesem Tage traten Vergiftungssymptome deutlich hervor. Am 16. Juli wurde das Tier durch Verbluten getötet. Der Hund hatte an Körpergewicht von $10 \mathrm{~kg}$ auf $8,3 \mathrm{~kg}$ abgenommen. Deutliche Fettleber.

Hund Nr. V erhielt am 6., 8., 10., 11., 13. und 14. Juni subkutane Injektionen von $0,5 \mathrm{ccm}$ Phosphoröl. Deutliche Wirkung (Depression, kein Appetit, Diarrhöe) am 14. VI. Plötzlicher Tod am 17. VI. Die Leber wurde eine halbe Stunde nach dem Tode mit gutem Erfolge mit 5 Liter Ringerscher Lösung durchspült. Deutliche Fettleber. Das Gewicht des Tieres hatte während der Vergiftungsperiode von $10,3 \mathrm{~kg}$ auf $8,9 \mathrm{~kg}$ abgenommen.

Sowohl die vergifteten wie die normalen Tiere wurden, mit Ausnahme von Nr. V, durch Verbluten getötet. Da es möglich erschien, daß der Blutgehalt der Leber einen Einfluß auf die gefundenen Werte der Hexonbasen ausübte, wusch ich in einzelnen Fällen (Nr. IV und V) die Leber blutfrei. Während das Tier Nr. IV durch die Carotis verblutete, wurde eine stetige Injektion warmer Ringerscher Lösung in die Vena jugularis gemacht und auf diese Weise mit Hilfe der Herztätigkeit des Tieres, die bekanntlich unter diesen Umständen ziemlich lange erhalten bleibt, das Blut größtenteils aus den Gefäßen herausgetrieben.

Hoppe-Seyler's Zeitschrift [. physiol. Chemie. XLIV. 
Die Leber wurde in allen Fällen zerkleinert, ein kleiner Teil zur Bestimmung des Wasser- und Stickstoffgehaltes entnommen und die Hauptmenge durch Kochen mit Schwefelsäure zerlegt. Hierbei schied sich ein unlöslicher fettiger Rückstand aus, dessen Stickstoffgehalt festgestellt und dem in der Lösung vorhandenen Stickstoff hinzu addiert wurde. ${ }^{1}$ ) Das Filtrat, in welchem sämtliche lösliche, durch die Spaltung der Lebersubstanz enthaltene Stoffe vorhanden waren, wurde nun nach den von A. Kossel und F. Kuts cher ${ }^{2}$ ) beschriebenen Methoden (mit der Modifikation von A. Kossel und A. J. Patten) ${ }^{3}$ ) verarbeitet.

Die Resultate sind in den folgenden Tabellen zusammengestellt.

Tabelle I.

\begin{tabular}{|c|c|c|c|c|c|c|c|c|c|c|}
\hline \multirow{2}{*}{$\begin{array}{c}\text { Ter- } \\
\text { couchs- } \\
\text { sulin- } \\
\text { mer }\end{array}$} & \multirow{2}{*}{$\begin{array}{c}\text { Ge- } \\
\text { wicht } \\
\text { der } \\
\text { Leber } \\
\text { g }\end{array}$} & \multirow{2}{*}{$\begin{array}{c}\text { Trocken- } \\
\text { substanz } \\
\text { in } 100 \\
\text { reilen des } \\
\text { feuchten } \\
\text { Organs }\end{array}$} & \multirow{2}{*}{$\begin{array}{c}\text { Stick- } \\
\text { stoff- } \\
\text { mengen } \\
\text { in 100 } \\
\text { Trilen } \\
\text { trocknen } \\
\text { Leber- } \\
\text { gewebes }\end{array}$} & \multicolumn{3}{|c|}{$\begin{array}{l}\text { Stickstoff der Basen } \\
\text { in } 100 \text { Teilen des } \\
\text { feuchten Gewebes }\end{array}$} & \multicolumn{3}{|c|}{$\begin{array}{c}\text { Stickstoff der } \\
\text { Basen in Pro- } \\
\text { zenten des } \\
\text { Gesamtstickstoffs }\end{array}$} & \multirow{2}{*}{$\begin{array}{l}\quad \mathrm{Be}- \\
\text { merkungen }\end{array}$} \\
\hline & & & & $\underset{\text { Arginin }}{\operatorname{im}}$ & $\operatorname{im}_{\text {Histidin }}$ & $\underset{\text { Lysin }}{i m}$ & $\operatorname{im}_{\text {Arginin }}$ & \begin{tabular}{|c|}
$\operatorname{im}_{\substack{\text { Histi- } \\
\text { din }}}$ \\
\end{tabular} & im & \\
\hline I. & 370 & 30,13 & 11,54 & 0,3121 & 0,0722 & 0,1694 & 8,982 & 2,077 & 4,876 & $\begin{array}{c}\text { Normales Tier. } \\
\text { Leber blut- } \\
\text { haltig }\end{array}$ \\
\hline II. & 435 & 28,50 & 11,28 & 0,3295 & 0,0774 & 0,1762 & 10,25 & 2,407 & 5,483 & \begin{tabular}{|} 
Normales Tier. \\
Leber blut- \\
haltig
\end{tabular} \\
\hline III. & 194 & 26,74 & 7,34 & 0,0991 & 0,0355 & 0,0759 & 5,046 & 1,809 & 3,868 & $\begin{array}{l}\text { Phosphorrergif- } \\
\text { tung. Leber } \\
\text { bluthaltig }\end{array}$ \\
\hline IV. & 180 & 22,59 & 12,48 & 0,2458 & 0,0673 & 0,1103 & 8,720 & 2,388 & 3,912 & $\begin{array}{c}\text { Normales Tier. } \\
\text { Leber ausge- } \\
\text { waschen }\end{array}$ \\
\hline $\mathrm{V}$. & 260 & 21,90 & 7,90 & 0,0717 & 0,0217 & 0,0469 & 4,143 & $1,2 \check{6} 6$ & 2,710 & $\begin{array}{l}\text { Phosphorvergif- } \\
\text { tung. Leber } \\
\text { ausgewaschen }\end{array}$ \\
\hline
\end{tabular}

1) Die Menge dieses in der unlöslichen Substanz enthaltenen Stickstoffs war in allen Fällen sehr gering.

2) Diese Zeitschrift, Bd. XXXI, S. 165.

:3) Diese Zeitschrift, Bd. XXXVIII, S. 39. 
Tabelle II.

In $100 \mathrm{~g}$ trocknen Lebergewebes:

\begin{tabular}{c|c|c|c|c}
\hline $\begin{array}{c}\text { Versuchs- } \\
\text { nummer }\end{array}$ & Arginin in $\mathbf{g}$ & Histidin in & Lysin in $\mathbf{g}$ & \\
\hline I. & 3,22 & 0,88 & 2,93 & Normal \\
\hline II. & 3,59 & 1,00 & 3,22 & Normal \\
\hline III. & 1,15 & 0,49 & 1,48 & Phosphorvergiftung \\
\hline IV. & 3,38 & 1,10 & 2,55 & Normal \\
\hline V. & 1,02 & 0,366 & 1,12 & Phosphorvergiftung
\end{tabular}

Es ergibt sich aus diesen Zahlen folgendes:

1. Die Lebersubstanz wird während der Phosphorvergiftung prozentisch ärmer an Stickstoff.

2. Unter dem Einfluß der Phosphorvergiftung nimmt die Menge des Arginins, Histidins und Lysins ab. Diese Annahme ist bemerkbar, wenn man die Menge des Basenstickstoffs auf das feuchte Organ bezieht (s. Tabelle I). Ebenso deutlich tritt sie in Tabelle II hervor, in welcher das Gewichtsverhältnis der Basen zum trockenen Lebergewebe dargestellt ist.

3. Die Menge des in den genannten Basen enthaltenen Stickstoffs nimmt im Vergleich zum gesamten Stickstoff der Leber bei der Phosphorvergiftung $a b$.

Die Hexonbasen werden also leichter als andere stickstoffhaltige Gruppen durch die der Vergiftung folgenden Auflösungsprozesse fortgeschafft.

Durch diese Untersuchungen wird zum erstenmal auf sicherer chemischer Grundlage festgestellt, in welcher Weise sich der Abbau der großen als "Eiweiß" bezeichneten Komplexe in dem der Degeneration anheimfallenden Gewebe vollzieht.

Der stickstoffreichste Teil des Eiweißmoleküls ist unter den von mir untersuchten Bedingungen der labilste. Dieser wird 
340. Wakeman, Über die chemische Veränderung der Leber etc.

infolge der durch Phosphorvergiftung veranlaßten Zersetzungsvorgänge zunächst fortgeschafft und es bleibt ein Rest, der ärmer an Stickstoff und an Basen ist.

Diese Resultate müssen die Anregung geben, auch andere degenerative Vorgänge in den Geweben in gleicher Weise zu untersuchen. In manchen Fällen wird man voraussichtlich den gleichen Verlauf des Eiweißzerfalls feststellen können. 